\title{
Utilização de plantas medicinais e fitoterápicos por pacientes submetidos à quimioterapia de um centro de oncologia de ljuí/RS
} The use of medicinal plants and herbal medicines for patients in chemotherapy in an oncology center of ljuí/RS

\author{
Gislaine Tisott Dal Molin* \\ Aline Williens Cavinatto* \\ Christiane de Fátima Colet ${ }^{* *}$
}

\begin{abstract}
Resumo
Pacientes oncológicos, procuram tratamentos alternativos e complementares em busca da melhora rápida, com destaque para as plantas medicinais e fitoterápicos. Este trabalho tem como objetivo verificar a prevalência de uso de plantas medicinais e fitoterápicos pelos usuários de um Centro de Alta Complexidade em Oncologia do Rio Grande do Sul (RS). Os dados foram coletados no Centro de Alta Complexidade em Oncologia (Cacon) do município de ljuí/RS, no período de junho a dezembro de 2013. Sendo incluídos todos os usuários que realizavam quimioterapia endovenosa. Foram entrevistados 130 pacientes, $62 \%$ do sexo feminino, com idade média de 56 anos. As plantas medicinais mais citadas foram Annona muricata (26\%), Aloe vera (23\%), Euphorbia tirucalli (8,8\%). Para as plantas mencionadas neste estudo, não foram encontradas referências que apresentassem evidências científicas de sua ação farmacológica para o tratamento do câncer, comprovando a falta de orientação dos pacientes sobre as plantas medicinais. Verificou-se que a indicação do uso de plantas medicinais, foi principalmente de familiares (68,5\%) e amigos (13,8\%), sem acompanhamento, orientação e conhecimento do médico. A falta de informações e de acompanhamento dos usuários pode ser um alerta aos órgãos sanitários, para melhorar a fiscalização e promover o desenvolvimento de estudos que evidenciem formas de utilização e os riscos das plantas medicinais.
\end{abstract}

Palavras chave: Espécies Vegetais. Neoplasia. Quimioterapia.

\begin{abstract}
Cancer patients seek to alternative and complementary treatments searching for rapid improvement, especially medicinal and herbal plant. This work objective is to evaluate the use of medicinal plants and herbal medicines in patients with cancer who are treated at the High Complexity in Oncology Center (CACON) in the city of ljuí/RS. Data were collected from June to December 2013. Being included all users who performed intravenous chemotherapy. It was interviewed 130 patients, $62 \%$ female, with a mean age of 56 years. The medicinal plants most frequent were Annona muricata (26\%), Aloe vera (23\%) Euphorbia tirucalli (8.8\%). For plants used in this study, there were no references to present scientific evidence of its pharmacological action for cancer treatment, proving the lack of education of the patients on medicinal plants. It was found that the indication of use of medicinal plants were mostly by family $(68.5 \%)$ and friends (13.8\%), without monitoring, guidance and medical knowledge. The lack of information and monitoring of users can be a warning to health authorities, to improve the oversight and promote the development of studies focusing on ways of its use and the risks of medicinal plants.
\end{abstract}

Keywords: Plant Species. Cancer. Chemotherapy.

DOI: 10.15343/0104-7809.20153903287298

\# Artigo resultante do trabalho de conclusão de curso: Gislaine Tisott Dal Molin.2014. Apresentada à Universidade Regional do Noroeste do Estado do Rio Grande do Sul, como parte das exigências para graduação em Farmácia.

* Universidade Regional do Noroeste do Estado do Rio Grande do Sul, Campus Ijuí, Departamento de Ciências da Vida, Ijuí, Rio Grande do Sul, Brasil.

** Universidade Regional do Noroeste do Estado do Rio Grande do Sul, Campus Ijuí, Departamento de Ciências da Vida, Ijuí, Rio Grande do Sul, Brasil. E-mail: Christiane.colet@unijui.edu.br

Os autores declaram não haver conflitos de interesse. 


\section{INTRODUÇÃO}

O uso de plantas medicinais é considerado uma prática influenciada pela crença popular, carência econômica, dificuldade de acesso à assistência médica/farmacêutica e pela mídia'. Elas representam uma forma de tratamento alternativo para diversas doenças, como por exemplo, o câncer. Os pacientes com esta doença, utilizam formas complementares ao tratamento convencional, entre as quais cita-se: homeopatia, espiritualidade, medicina religiosa e o reiki, com destaque à utilização da fitoterapia. Estes podem complementar os convencionais que são: a quimioterapia, a radioterapia, a hormonoterapia, a imunoterapia e a intervenção cirúrgica² .

Segundo Cassileth e Chapman ${ }^{3}$, a maior parte dos pacientes faz uso de práticas complementares e alternativas, sem que os médicos sejam informados. As razões são diversas, dentre as quais destaca-se: percepção da falta de interesse do médico em ouvir sobre o tema, a antecipação de uma reação negativa por parte deste profissional e a crença de que os médicos não possuem conhecimento e treinamento acerca de medicinas alternativas. Desta forma, a busca por outras alternativas esta relacionada com reclamações dos pacientes quanto a um relacionamento insensível, limitado e apressado com os oncologistas.

Para dar aporte às ações relacionadas ao conhecimento, uso e ações de plantas medicinais o governo brasileiro publicou legislações relacionadas ao tema como: Programa Nacional de Plantas Medicinais e Fitoterápicos ${ }^{4}$; Resolução $n^{\circ}$ 10/2010 ; Formulário de Fitoterápicos da Farmacopeia Brasileira ${ }^{6}$. Um dos objetivos das legislações supracitadas foi monitorar e avaliar os produtos utilizados pela população, visando o uso seguro e racional destas terapias, amparado por pesquisas científicas e orientado por profissionais da saúde, além da educação continuada dos profissionais da saúde sobre esta temática.

Mesmo com essas políticas, levantamentos realizados em bases de dados nacionais mostram que existem poucas pesquisas acerca deste tema. Um artigo encontrado aborda a utilização de plantas medicinais por pacientes oncológicos e familiares em um centro de radioterapia, de uma Universidade do Rio Grande do Sul 7 , contudo este estudo foca em pacientes submetidas a radioterapia, diferindo do presente estudo. Outros estudos encontrados com esta temática foram: Araújo, et al., 2007ं Casarin, et al., 2005²; Diaz, et al., 2012 ${ }^{9}$; Elias e Alves, $2002^{10}$ que serão abordados ao longo do estudo, mas em locais e particularidades distintas desta pesquisa.

Neste contexto, os estudos epidemiológicos podem favorecer o uso racional das terapias, ao buscar conhecer os hábitos de uso das plantas medicinais e fitoterápicos, e com esses dados subsidiar a formulação de programas educacionais que ofereçam orientação sobre segurança no autocuidado com a utilização de diferentes terapias, identificando interações dos fitoterápicos e/ou medicamentos ${ }^{11}$. E ainda, para auxiliar os profissionais de saúde a incorporar as plantas medicinais nativas como recurso terapêutico ${ }^{12}$.

Diante do exposto, o objetivo deste trabalho foi verificar a prevalência de uso de plantas medicinais e fitoterápicos pelos usuários de um Centro de Alta Complexidade em Oncologia do Rio Grande do Sul (RS).

\section{MÉTODO}

Trata-se de estudo transversal realizado com aplicação de questionário estruturado, quali-quantitativo, em usuários do Centro de Alta Complexidade de Oncologia (Cacon) de um hospital de nível IV do município de ljuí/RS. Foram selecionados usuários que realizavam quimioterapia endovenosa neste local, de ambos os sexos e maiores de 18 anos de idade. Os dados foram coletados no período de junho a dezembro de 2013.

Segundo informações obtidas no local de estudo são realizadas 12 sessões de quimioterapia por via endovenosa, em cada turno, perfazendo um total de 430 pacientes distintos por mês. Este dado foi utilizado para o cálculo amostral e obteve-se uma amostra de 130 pacientes, com erro amostral de 5\% e nível de significância de $90 \%$. 
A coleta de dados foi realizada nos dias e turnos aleatórios, escolhendo os entrevistados por sorteio das cadeiras em cada turno. $\mathrm{O}$ instrumento de coleta dos dados foi, preenchidos pelos pesquisadores de acordo com as respostas dos entrevistados.

As plantas medicinais e fitoterápicas citadas pelos entrevistados foram avaliadas quanto a segurança e a eficácia conforme a Instrução Normativa $n^{0}$ 5, de 31 de março de 2010, que estabelece a lista de referências bibliográficas para avaliação destes produtos, sendo que para esse trabalho foram utilizadas as referências indicada no grupo $A^{13}$. Além disso, foram consultados: Formulário de Fitoterápicos da Farmacopeia Brasileira6; Resolução n 10/2010, Anexo I5; Relação Nacional de Medicamentos Essenciais (RENAME) ${ }^{14}$; Farmacognosia: da planta ao medicamento ${ }^{15}$.

Este estudo foi aprovado pelo Comitê de Ética da Universidade Regional do Noroeste do Estado do Rio Grande do Sul através do Parecer Consubstanciado no. 298.367/2013.

\section{RESULTADOS}

Foram entrevistados 130 usuários, sendo $62 \%$ do sexo feminino. A média da idade dos pacientes foi de 56,86 anos ( $\mathrm{DP} \pm 11,61)$, com idade mínima de 28 anos e a máxima de 88 anos, sendo a faixa etária mais prevalente entre 60 a 69 anos. Sobre a escolaridade dos usuários, $55,4 \%$ possuíam ensino fundamental incompleto e $66 \%$ eram casados. Dos entrevistados $34 \%$ residiam na zona rural dos municípios de origem e apenas 13\% moravam no município de ljuí/RS; já 10\% residiam de Santo Ângelo/RS e os demais em 53 municípios pertencentes a $4^{\circ}, 12^{\circ}, 15^{\circ}$, $17^{\circ}$ e $19^{\circ}$ Coordenarias Regionais de Saúde do Estado do Rio Grande do Sul. Quanto a profissão dos entrevistados 30,8\% eram aposentados e recebiam uma renda média de 1,6 salários mínimos (DP $\pm 1,4)$, sendo que $51 \%$ declararam receber um salário mínimo.

Quando questionados sobre os problemas de saúde, além do câncer, para o qual os mesmos estavam realizando tratamento, os pacientes citaram 17 doenças distintas, com média de
0,81 (DP $\pm 1,0)$ doenças por entrevistado, sendo todas consideradas problemas crônicos de saúde. Entre os entrevistados, 65 informaram não possuir nenhuma doença, 40 afirmaram ter uma doença, 14 citaram duas doenças e 11 três ou mais doenças, e o máximo citado foram de cinco doenças por usuário. Além disso, 35\% apresentavam hipertensão arterial sistêmica (HAS). Alguns desses dados encontram-se descritos na Tabela 1.

A média de tempo de quimioterapia dos entrevistados foi de 22 meses (DP $\pm 30,99)$, tendo pacientes que tinham começado no dia da entrevista e o tempo máximo foi de 192 meses. O câncer que mais prevaleceu entre os pacientes foi o de mama $(32,3 \%)$, seguido pelo de intestino $(16,1 \%)$ e o de pulmão $(11,5 \%)$, como pode ser observado na Tabela 1. Em relação ao estadiamento do câncer observou-se que $24.6 \%$ dos entrevistados encontravam na fase três do tumor e 56\% apresentam metástase na fase zero.

Todos os pacientes entrevistados faziam uso de plantas medicinais, destes $67 \%$ as utilizam em busca de uma ação terapêutica e os demais apenas como bebida. Especificamente para o tratamento adjuvante do câncer o uso de plantas medicinais foi relatado por $40 \%$ da população em estudo. Ao serem questionados sobre os benefícios das plantas medicinais e fitoterápicos à saúde, 94\% acreditavam que os produtos à base de plantas fazem bem a saúde.

Foram citadas para uso geral de tratamento de saúde 316 plantas, pertencentes a 72 espécies distintas, com média de 2,4 (DP $\pm 1,30)$ plantas por paciente.

Os entrevistados foram questionados quanto a procedência das plantas medicinais utilizadas, a maioria (69\%) informou que as mesmas eram cultivadas no quintal das próprias residências, $36 \%$ compradas em estabelecimentos farmacêuticos, já 30\% eram obtidas em supermercados. Ao serem questionados sobre mistura de plantas medicinais, 90\% relataram que faz uso de apenas uma única planta medicinal em cada utilização.

A utilização das plantas e a frequência da utilização das mesmas podem ser visualizados na Tabela 2. 
Tabela 1. Caracterização socioeconômica e tipo de câncer dos entrevistados submetidos à quimioterapia de um centro de oncologia de ljuí/RS. n=130, 2013.

\begin{tabular}{lcc}
\hline Características socioeconômicas & $\mathbf{n}$ & $\%$ \\
\hline Sexo & & \\
\hline Feminino & 81 & 62 \\
Masculino & 49 & 38 \\
\hline Escolaridade & & \\
\hline Ensino fundamental incompleto & 72 & 55,4 \\
Ensino médio completo & 19 & 14,6 \\
Ensino superior completo & 15 & 11,5 \\
Analfabeto & 10 & 7,8 \\
Ensino fundamental completo & 8 & 6,1 \\
Ensino médio incompleto & 5 & 3,8 \\
Ensino superior incompleto & 1 & 0,8 \\
\hline
\end{tabular}

\begin{tabular}{l} 
Profissão \\
\hline Aposentado (a) \\
Agricultor (a) \\
Do lar \\
Professor (a) \\
Doméstica \\
Comerciário (a) \\
Funcionário (a) público (a) \\
Outros
\end{tabular}

\begin{tabular}{l} 
Tipo de câncer \\
\hline Mama \\
Intestino \\
Pulmão \\
Útero \\
Ovário \\
Outros $^{2}$
\end{tabular}

\section{Estadiamento do tumor}

\begin{tabular}{lcc}
\hline Fase 3 & 32 & 24,6 \\
Fase 0 & 31 & 23,9 \\
Fase 1 & 26 & 20 \\
Fase 4 & 24 & 18,5 \\
Fase 2 & 17 & 13 \\
\hline Estadiamento da metástase & & 56 \\
\hline Fase 0 & 73 & 44 \\
Fase 1 & 57 & 23 \\
\hline
\end{tabular}

${ }^{1}$ Motorista; Empresário; Camareira; Agente de Saúde; Artesã; Pedreiro; Maquinista; Publicitário; Ajudante de produção; Gerente de loja; Metalúrgico; Serviços gerais; Manicure; Telefonista; Vigilante.

${ }^{2}$ Ossos; Bexiga; Fígado; Leucemia; Esôfago; Pâncreas; Pele; Próstata; Garganta; Laringe; Cabeça e pescoço; Bile; Abdome; Glândulas linfáticas; Linfoma de Hodgkin. 
Tabela 2. Caracterização do uso de plantas medicinal e fitoterápico na forma de chás por usuários submetidos à quimioterapia de um centro de oncologia de ljuí/RS, 2013.

\begin{tabular}{|c|c|c|}
\hline Uso das plantas & $\mathbf{N}$ & $\%$ \\
\hline \multicolumn{3}{|c|}{ Utilização com intuito de tratar o câncer $(n=130)$} \\
\hline Não & 78 & 60 \\
\hline Sim & 52 & 40 \\
\hline \multicolumn{3}{|c|}{ As plantas medicinais trazem benefícios a Saúde $(n=130)$} \\
\hline Sim & 123 & 94 \\
\hline Não & 7 & 6 \\
\hline \multicolumn{3}{|c|}{ As plantas medicinais podem fazer mal a Saúde $(n=130)$} \\
\hline Não & 98 & 75 \\
\hline Sim & 32 & 25 \\
\hline \multicolumn{3}{|l|}{ Como é a planta que é utilizada ( $n=179$ ) } \\
\hline Planta fresca & 109 & 61 \\
\hline Sachê & 56 & 31,2 \\
\hline Cápsula & 7 & 4 \\
\hline Pomada & 4 & 2,2 \\
\hline Xarope & 3 & 1,6 \\
\hline \multicolumn{3}{|l|}{ Usa fitoterápico com prescrição médica $(n=7)$} \\
\hline Não & 7 & 100 \\
\hline $\operatorname{Sim}$ & 0 & 0 \\
\hline \multicolumn{3}{|l|}{ Quem indicou as plantas medicinais $(n=130)$} \\
\hline Família & 89 & 68,5 \\
\hline Amigos & 18 & 13,8 \\
\hline Vizinho & 6 & 4,6 \\
\hline Internet & 5 & 3,8 \\
\hline Livros & 3 & 2,4 \\
\hline Outros $^{1}$ & 9 & 6,9 \\
\hline Total: & 130 & 100 \\
\hline \multicolumn{3}{|c|}{ Frequência do uso de plantas medicinais ( $n=130$ ) } \\
\hline Raramente (somente quando se sente mal) & 65 & 50 \\
\hline Frequentemente ( $4 \mathrm{X}$ ou mais por semana) & 60 & 46 \\
\hline Pouca utilização & 5 & 4 \\
\hline Total: & 130 & 100 \\
\hline
\end{tabular}

${ }^{1}$ Mídia; Vendedora; Massoterapeuta; Pastor; Agente de Saúde; Funcionários do Cacon; Professor; Médico; Nutricionista.

Conforme Tabela 3, foram citadas 68 plantas medicinais, com repetições, e 13 plantas distintas, estas foram utilizadas especificamente para o tratamento do câncer. As plantas medicinais mais prevalentes foram: a Annona muricata (graviola) utilizada em cápsulas e em infusão, seguida pela Aloe vera (babosa), esta foi citada como uso em "garrafada", contendo babosa, mel e cachaça, utilizando uma colher de sopa por três vezes ao dia, ressalta-se que os pa- 
cientes que utilizam este último produto, três o compram pronto de ambulantes e armazenam na geladeira, fazendo uso por uma semana, os demais produziam a garrafada em seu próprio domicilio e também a armazenavam na geladeira. O Euphorbia tirucalli (avelós) também foi citado por seis entrevistados e utilizava-se duas gotas do látex na alimentação regularmente.

Tabela 3. Plantas medicinais e fitoterápicas citadas para o câncer pelos pacientes submetidos à quimioterapia de um centro de oncologia de ljuí/RS. n=68, 2013.

\begin{tabular}{|c|c|c|c|c|c|c|}
\hline $\begin{array}{l}\text { Nome } \\
\text { Científico/ } \\
\text { Nome Popular }\end{array}$ & $\begin{array}{c}n \\
(\%)\end{array}$ & $\begin{array}{l}\text { Parte da } \\
\text { planta (n) }\end{array}$ & $\begin{array}{l}\text { Forma de } \\
\text { preparo }(n)\end{array}$ & $\begin{array}{l}\text { Tipo de } \\
\text { Câncer }\end{array}$ & Indicação da Literatura & $\begin{array}{c}\text { De acordo } \\
\text { /Desacordo }\end{array}$ \\
\hline $\begin{array}{l}\text { Annona muricata } \\
\text { Graviola }\end{array}$ & $\begin{array}{c}18 \\
(26 \%)\end{array}$ & $\begin{array}{l}\text { Folha (13) } \\
\text { Cápsula (5) }\end{array}$ & $\begin{array}{l}\text { Infusão (12) } \\
\text { Deccoção (1) }\end{array}$ & $\begin{array}{c}\text { Mama (9); } \\
\text { Útero (3); } \\
\text { Pâncreas (2); } \\
\text { Pulmão (2); } \\
\text { Estomago (1); } \\
\text { Pele (1). }\end{array}$ & $\begin{array}{l}\text { Não encontrado nas referências } \\
\text { pesquisadas. }\end{array}$ & Desacordo \\
\hline
\end{tabular}

\begin{tabular}{|c|c|c|c|c|c|c|}
\hline $\begin{array}{l}\text { Aloe vera } \\
\text { Babosa }\end{array}$ & $\begin{array}{c}16 \\
(23 \%)\end{array}$ & Folha (16) & Garrafada (16) & $\begin{array}{l}\text { Mama (6); } \\
\text { Intestino (4); } \\
\text { Pulmão (3); } \\
\text { Garganta (1); } \\
\text { Esôfago (1); } \\
\text { Próstata (1). }\end{array}$ & $\begin{array}{l}\text { Uso externo: Cicatrizante } \\
\text { Tratamento tópico de } \\
\text { queimaduras de } 1^{\circ} \text { e } 2^{\circ} \text { graus } \\
\text { e como coadjuvante nos } \\
\text { casos de Psoríase vulgaris }{ }^{\mathrm{C}, \mathrm{E}, \mathrm{F}} \text {; } \\
\text { Anti-inflamatório, emoliente, } \\
\text { hidratante, e antibacteriana }{ }^{\mathrm{C}, \mathrm{D}, \mathrm{F}} \text {. }\end{array}$ & Desacordo \\
\hline
\end{tabular}

\begin{tabular}{lccc}
\hline Matricariarecutita & 7 & Gel (3) & Uso externo (3) \\
Camomila & $(10,2 \%)$ & Folha (3) & Infusão para \\
& & Semente (1) & enxaguar a \\
& & boca após a \\
& & quimioterapia (3) \\
& & Decocção para \\
& & fazer compressas
\end{tabular}

Intestino (2); Uso interno: Antispasmódico, Cabeça e ansiolítico e sedativo leve;

Desacordo pescoço (1); Dispepsia, flatulência Gânglios epigástrica, digestão linfáticos (1); prejudicada, e flatulência. Mama (2); $\quad$ Calmante e em casos Útero (1). $\quad$ suaves de insônia devido a distúrbios do sistema nervoso ${ }^{\mathrm{D}}$; Hipoglicemiante; Hipolipemiante $^{\mathrm{A}, \mathrm{B}}$; Dispepsia, como colagogo e colerético ${ }^{\mathrm{B}}$. Uso externo: Anti-inflamatório em afecções da cavidade oral ${ }^{\mathrm{A}}$; Inflamação e irritações da pele e mucosas (rachaduras da pele, hematomas, queimaduras e picadas de insetos), incluindo irritações e infecções da boca e gengivas, e hemorróidas ${ }^{\mathrm{c}}$.

\begin{tabular}{|c|c|c|c|c|c|c|}
\hline $\begin{array}{l}\text { Euphorbia } \\
\text { tirucalli } \\
\text { Avelós }\end{array}$ & $\begin{array}{c}6 \\
(8,8 \%)\end{array}$ & $\begin{array}{l}\text { Látex (5) } \\
\text { Folha (1) }\end{array}$ & Infusão (1) & $\begin{array}{l}\text { Mama (2); } \\
\text { Pâncreas (1); } \\
\text { Pulmão (1); } \\
\text { Leucemia (1); } \\
\text { Útero (1). }\end{array}$ & $\begin{array}{l}\text { Não encontrado nas referências } \\
\text { pesquisadas. }\end{array}$ & Desacordo \\
\hline $\begin{array}{l}\text { Malva officinalis } \\
\text { Malva }\end{array}$ & $\begin{array}{c}4 \\
(5,8 \%)\end{array}$ & $\begin{array}{l}\text { Folha (2) } \\
\text { Sache (2) }\end{array}$ & $\begin{array}{c}\text { Infusão para } \\
\text { enxaguar a } \\
\text { boca após a } \\
\text { quimioterapia (4) }\end{array}$ & $\begin{array}{c}\text { Mama }(1) ; \\
\text { Intestino }(1) ; \\
\text { Cabeça e } \\
\text { pescoço }(1) ; \\
\text { Ossos }(1)\end{array}$ & $\begin{array}{l}\text { Uso interno: Expectorante }{ }^{\mathrm{A}} \text {; } \\
\text { Uso externo: Anti-inflamatório } \\
\text { e antisséptico da cavidade } \\
\text { oral }^{\mathrm{A}} \text {; Forma uma camada } \\
\text { protetora sobre a cavidade } \\
\text { oral, da mucosa da faringe, } \\
\text { calmante na irritação local e } \\
\text { anti-inflamatória }{ }^{\mathrm{C}} \text {. }\end{array}$ & Desacordo \\
\hline $\begin{array}{l}\text { Maytenus } \\
\text { officinalis } \\
\text { Espinheira Santa }\end{array}$ & $\begin{array}{c}3 \\
(4 \%)\end{array}$ & Folhas (2) & $\begin{array}{c}\text { Infusão (2) } \\
\text { Decocção (1) }\end{array}$ & $\begin{array}{l}\text { Útero (2); } \\
\text { Mama (1). }\end{array}$ & $\begin{array}{l}\text { Uso interno: Antidispéptico, } \\
\text { antiácido e protetor do muco }{ }^{\mathrm{A}} ; \\
\text { Coadjuvante no tratamento de } \\
\text { gastrite e úlcera gastroduodenal } \\
\text { e sintomas dispepsia }{ }^{\mathrm{E}} \text {. }\end{array}$ & Desacordo \\
\hline
\end{tabular}


...continuação

\begin{tabular}{|c|c|c|c|c|c|c|}
\hline $\begin{array}{l}\text { Nome } \\
\text { Científico/ } \\
\text { Nome Popular }\end{array}$ & $\begin{array}{c}n \\
(\%)\end{array}$ & $\begin{array}{l}\text { Parte da } \\
\text { planta (n) }\end{array}$ & $\begin{array}{l}\text { Forma de } \\
\text { preparo }(n)\end{array}$ & $\begin{array}{l}\text { Tipo de } \\
\text { Câncer }\end{array}$ & Indicação da Literatura & $\begin{array}{l}\text { De acordo } \\
\text { /Desacordo }\end{array}$ \\
\hline $\begin{array}{l}\text { Agaricusblazei } \\
\text { Murrill } \\
\text { Cogumelo do sol }\end{array}$ & $\begin{array}{c}2 \\
(3 \%)\end{array}$ & Cápsula (2) & & Mama (2). & $\begin{array}{l}\text { Não encontrado nas referências } \\
\text { pesquisadas. }\end{array}$ & Desacordo \\
\hline $\begin{array}{l}\text { Citrus limon } \\
\text { Limão }\end{array}$ & $\begin{array}{c}2 \\
(3 \%)\end{array}$ & Fruto (2) & $\begin{array}{l}\text { Ralado na } \\
\text { comida (2) }\end{array}$ & $\begin{array}{l}\text { Mama (1); } \\
\text { Pele (1). }\end{array}$ & $\begin{array}{l}\text { Não encontrado nas referências } \\
\text { pesquisadas. }\end{array}$ & Desacordo \\
\hline $\begin{array}{l}\text { Equisetum } \\
\text { Arvense } \\
\text { Cavalinha }\end{array}$ & $\begin{array}{c}2 \\
(3 \%)\end{array}$ & $\begin{array}{l}\text { Folha (1) } \\
\text { Talo (1) }\end{array}$ & Decocção (2) & $\begin{array}{l}\text { Mama (1); } \\
\text { Intestino (1). }\end{array}$ & $\begin{array}{l}\text { Uso interno: Edemas por } \\
\text { retenção de líquidos }{ }^{\mathrm{B}} \text {. }\end{array}$ & Desacordo \\
\hline $\begin{array}{l}\text { Allium Sativum } \\
\text { Alho }\end{array}$ & $\begin{array}{c}1 \\
(1,5 \%)\end{array}$ & $\begin{array}{l}\text { Dente de } \\
\text { alho (1) }\end{array}$ & & Mama (1). & $\begin{array}{l}\text { Uso interno: Anti-hipertensivo, } \\
\text { auxilia nos sintomas de gripes } \\
\text { e resfriados e auxilia na } \\
\text { prevenção da aterosclerose }{ }^{\mathrm{C}, \mathrm{D}, \mathrm{F}} \text {; } \\
\text { Hipercolesterolemia, atua como } \\
\text { expectorante e anti-séptico }{ }^{\mathrm{B}} \text {; } \\
\text { Antibacteriana, antimicótica, } \\
\text { antiviral, antiparasitária, } \\
\text { anti-inflamatório, atuam } \\
\text { no sistema imunológico, } \\
\text { antioxidante e fibrinolítica, } \\
\text { além de inibição da agregação } \\
\text { plaquetária e diminuição } \\
\text { das taxas de triglicerídeos e } \\
\text { colesterol }^{\mathrm{D}} \text { e lipídios } \text { lif. }^{\mathrm{F}}\end{array}$ & Desacordo \\
\hline $\begin{array}{l}\text { Peumus boldus } \\
\text { Boldo }\end{array}$ & $\begin{array}{c}1 \\
(1,5 \%)\end{array}$ & Folha (1) & Infusão (1) & Mama (1). & $\begin{array}{l}\text { Dispepsia (distúrbios da } \\
\text { digestão), como colagogo e } \\
\text { colerético }^{A, B} \text {. }\end{array}$ & Desacordo \\
\hline Outros ${ }^{1}$ & $\begin{array}{c}6 \\
(10,2 \%)\end{array}$ & & & & & \\
\hline
\end{tabular}

A - Formulário de Fitoterápicos da Farmacopéia Brasileira, 2011; B - Anexo I da RDC n 10/2010; C - WORLD HEALTH ORGANIZATION. WHO monographs on selected medicinal plants. Geneva, Switzerland: World Health Organization, 1999. v.1; v.2; v.3; v.4; D - SIMÕES, C.M.O.; SCHENKEL, E.P.; GOSMAN, G. et al. (Org.) Farmacognosia: da planta ao medicamento. 6 ed. Porto Alegre: Editora da UFSC e UFRGS Editora., 2007; E - RENAME, 2013; F - EUROPEAN SCIENTIFIC COOPERATIVE ON PHYTOTHERAPY (ESCOP). Monographs: The Scientific Foundation for Herbal Medicinal Products. 2 ed. Exeter, UK: European Scientific Cooperative on Phytotherapy and Thieme, 2003. ${ }^{1}$ muricata $^{\circledR}$ e ipê roxo

\section{DISCUSSÃO}

No presente estudo prevaleceu o sexo feminino, de forma semelhante ao estudo realizado em ambulatórios e em enfermarias de dois Hospitais, um universitário e outro filantrópico, localizados no município de João Pessoa/PB, entre agosto e 2000 a junho de 2001, no qual entre os 40 pacientes de câncer entrevistados 25 eram do sexo feminino ${ }^{8}$. Os dados destes estudos assemelham-se também ao presente estudo quanto a escolaridade e renda.

Sobre a utilização de plantas medicinais $40 \%$ dos pacientes do Cacon relataram consumir com intuito de tratar o câncer e no estudo de Araújo
$(2007)^{8}$ este valor foi de $47,5 \%$, na pesquisa de Casarin e colaboradores $(2005)^{2}$ foi de $57 \%$, sendo que este último estudo abordou tratamentos alternativos, informando que houve predomínio da fitoterapia, contudo sem informar a porcentagem específica desta prática. Já na pesquisa realizada no ambulatório no Centro Javeriano de Oncologia do Hospital Universitário San Ignácio, em Bogotá/Colômbia, entre junho e dezembro de 2011, relatou-se que dos 404 pacientes entrevistados, $77 \%$ utilizavam terapias à base de plantas ${ }^{9}$. Já no estudo de Jacondino, Amestoy, Thofehrn (2008) $)^{16}$ evidenciou-se que 89\% dos entrevistados conhecem algum tipo 
de terapia alternativa, sendo a fitoterapia a mais citada 38\%. Estes dados demonstram que o uso de fitoterápicos é uma prática bastante comum entre os pacientes oncológicos, sendo evidenciada em todos os estudos citados. Esta prática pode ser complementar, mas em algumas vezes, pode ser substitutiva ao tratamento convencional, mostrando a necessidade de conhecimento de plantas medicinais e fitoterápicos pelos pacientes, bem como um acompanhamento e orientação visando um uso seguro.

Sobre o estudo de Casarin e colaboradores $(2005)^{2}$, realizado no setor de oncologia de um hospital de médio porte da região sul do Brasil, com 30 entrevistados, os fitoterápicos comumente utilizados pelos pacientes foram a Aloe vera (babosa), o Agaricusblasei (cogumelo do Sol) e o Euphorbia entheurodoxalinneo (avelós). Essas três plantas também foram citadas no presente estudo, contudo diferindo a frequência.

No estudo realizado em Centro Javeriano de Oncologia com pacientes com câncer de mama, sobre a utilização das plantas que mais prevaleceu foram a Petiveria alliacea (namú), Aloe vera (babosa) e Annona muricata (graviola) ${ }^{9}$. Apenas a Petiveria alliacea não foi citada no presente estudo. Observou-se que na população dos três estudos as plantas medicinais mais prevalentes para tratamento complementar do câncer foram as mesmas, mesmo não sendo plantas nativas do local de estudo, observando um uso tradicional de plantas medicinais é difundido entre as populações. Destaca-se que as plantas medicinais que foram citadas em todos estudos deveriam ser melhor pesquisadas a partir de estudos pré-clínicos e clínicos para subsidiar este uso tradicional e trazer informações acerca da segurança do uso, que possam dar aporte de informações aos programas de educação continuada dos profissionais da saúde que trabalham nessa área.

A Annona muricata, segundo as referências pesquisadas, não apresentou comprovação científica de ação farmacológica para o tratamento do câncer. $\mathrm{O}$ que pode ser um indicativo da falta de estudos sobre sua ação e/ou seus efeitos indesejávis para a saúde da população. Além

disso, a Resolução no 2.744, de 16 de junho de $2010^{17}$, determinou a suspensão em todo o território nacional, das propagandas de produtos de graviola, devido aos mesmos não possuírem registro na Agência Nacional de Vigilância Sanitária (ANVISA) e por não haverem estudos científicos que comprovam a sua ação terapêutica para tal tratamento. Mostrando que embora o uso seja frequente entre os pacientes, esta planta pode desencadear riscos ao serem utilizadas e mostrando a necessidade de orientação destes pacientes acerca das terapias complementares que os mesmos vêm fazendo uso.

Sobre a Aloe vera (babosa), nenhuma referência indicava, sua utilização por via oral. Além disso, a ANVISA institui uma normativa $n^{\circ} .47$, de 16 de novembro de 2011, sobre o uso desta planta por via oral, afirmando que a mesma não pode ser ingerida, considerando não haver comprovação de segurança e não possuir produtos registrados à base de Aloe vera para uso interno. Esta normativa também argumenta que não existem estudos toxicológicos adequados e falta padronização ou especificação dos mesmos. Diante do exposto, a Aloe vera é recomendada apenas para uso externo ${ }^{18}$. Verificou-se no estudo, ao contrário, a utilização oral pelos pacientes entrevistados, que pode estar relacionado a falta de orientação quanto ao uso, podendo gerar riscos à saúde dos usuários, sendo este mais grave em pacientes oncológico submetido a quimioterapia que são pacientes suscetíveis, para os quais um tratamento inadequado pode gerar prejuízos mais pronunciados e prejudicar, inclusive, o tratamento contra o câncer.

Ainda sobre Aloe vera, estudos indicam que o uso interno prolongado pode provocar perda de minerais essenciais, sendo que a utilização diária deste preparado, por períodos extensos, por conter antraquinonas como grupo químico, pode provocar dores abdominais, diarreia sanguinolenta, hemorragias gástricas e nefrite. Os sintomas de intoxicação por consumo excessivo do látex são basicamente: hipocalemia, hiperaldosteronismo, pulso lento e hipotermia. O sintoma mais importante nos quadros de intoxicação é a diarreia ${ }^{18}$. Estes dados são bastante 
importantes considerando que pacientes oncológicos, em quimioterapia, já estão sujeitos aos efeitos colaterais destes tratamentos, que podem ser potencializados pelos efeitos colaterais da Aloe vera.

O Euphorbia tirucalli (avelós), também não foi encontrado, nas referências pesquisadas, nenhuma indicação para o mesmo. A ANVISA proibiu pela Resolução n² 2.917 de julho de 2011, a comercialização e a distribuição de produtos a base de avelós, por não ter sido submetida a testes de segurança e eficácia nesta Agência. Destacam-se como principais riscos da sua seiva branca, como o látex, irritação na pele e nas membranas mucosas, além de poder causar inflamação de pele, conjuntivite, queimaduras orais e na garganta, diarreia e gastroenterite ${ }^{19}$. Embora haja uma contraindicação para o uso, desde 2011, verificou-se que os pacientes, utilizam o látex, baseado no conhecimento popular, sem orientação de profissional da saúde.

Outra planta medicinal também citada com menos prevalência pelos entrevistados foi o Agaricusblazei Murrill (Cogumelo do sol), este produto apresenta registro na ANVISA somente como suplemento alimentar, não apresentando indicação para fim terapêutico de tratamento oncológico $^{17}$.

Observou-se neste estudo que para nenhuma das plantas medicinais mencionadas, foram encontradas referências que apresentassem evidências científicas de sua ação farmacológica para o tratamento do câncer. Isso comprova a falta de orientação dos pacientes sobre as plantas medicinais, sendo este fato agravado pela maioria dos entrevistados acreditarem que as plantas medicinais fazem somente bem a saúde. Ainda, verificou-se que as plantas medicinais foram indicadas por familiares, amigos ou vizinhos, sem acompanhamento, orientação e conhecimento do médico. Com ausência de confirmação das espécies, sem embasamento cientifico verificado neste estudo.

A indicação do uso de plantas por familiares e amigos, que foi evidenciada em nosso estudo, também foi verificada em pesquisa realizada com os pais das crianças com câncer atendidas no Serviço de Oncologia Pediátrica do Hospital Universitário da Universidade de Brasília/DF, no período de março a agosto de 2002. Este estudo mostrou que 33\% informaram que a indicação do uso de plantas concomitante a quimioterapia, foram realizadas por pessoas conhecidas e leigas ${ }^{10}$. Em nosso estudo, foram encontrados dados semelhantes, sobre as indicações de plantas medicinais para o tratamento de câncer, prevalecendo às pessoas mais próximas do paciente, como os familiares.

Para Jacondino $(2008)^{16}$ muitos pacientes oncológicos e suas famílias ao defrontar-se com a gravidade de sua doença buscam suporte físico e emocional de diversas formas, na esperança de cura através do tratamento alopático, porém, este método desencadeia efeitos adversos desagradáveis, como a queda dos cabelos, náuseas e vômitos, entre outros. Isto acaba incentivando o uso de outras medidas terapêuticas a fim de auxiliar à terapêutica convencional.

No presente estudo a maioria da população acreditava que as plantas não traziam malefício a saúde e não seria necessária comprovação de estudo para o uso, dado este que está correlacionado com o fato do uso de plantas ser baseado no conhecimento tradicional, sem orientação dos profissionais da saúde. Isso vai ao encontro do dado levantado no estudo de Araújo e colaborares $(2007)^{8}$ no qual a maioria dos pacientes não buscava informação cientifica sobre plantas (70\%), acreditando que "se bem não fizer, mal não fará". De forma complementar Cararin e colaboradores $(2005)^{2}$, observou-se que parte os pacientes e seus familiares optam pelo tratamento alternativo ou complementar, mesmo sem a indicação ou a aprovação do médico ou de outro profissional da saúde, por acreditarem que estes trazem benefícios à saúde. Além disso, pelo fato da população deste estudo ter apresentado nível de escolaridade baixo, as informações científicas podem ser de difícil acesso ou entendimento para os mesmos, necessitando de auxílio dos profissionais da saúde para tal. O que nesse estudo não ocorreu, na maioria dos casos, visto que, como mencionado, a indicação ocorreu principalmente por familiares e amigos 
que, muitas vezes, não possuíam conhecimento científico validado sobre o assunto.

Por outro lado, a maioria dos entrevistados afirmou não relatar a utilização de plantas medicinais aos médicos no momento da consulta, justificando que este profissional nunca questiona sobre práticas alternativas, ou alguns acreditam que o médico possa ser contra o uso. $\mathrm{Na}$ dos entrevistados afirmaram que não informam para seu médico a utilização de plantas medicinais, sendo relatado, da mesma forma, que os médicos não perguntam sobre a utilização ou julgam essa informação irrelevante. A falta de troca de informação entre paciente e médico pode agravar a saúde do paciente, colocando-a em risco. A omissão de informações por parte do usuário ao médico ocorre pelo fato destes julgarem que os médicos não aceitariam o uso, pela falta de estudos comprovando a eficácia de plantas medicinais para a cura do câncer. Contudo, essa comunicação deveria ser clara, permitindo ao médico direcionar e orientar o uso concomitante de tratamentos alternativos, quando julgar necessário ${ }^{20}$.

Em suma, o paciente tem o direito de ser orientado quanto ao mecanismo de ação das diferentes tratamentos terapêuticos não-convencionais, sua eficácia, indicações, contraindicações, custo, procedência de produtos e efeitos adversos possíveis, assim como do risco de interações. E os profissionais da saúde deve ter uma atitude de neutralidade quanto ao uso da medicina alternativa, compreendendo a diversidade cultural, social e étnica do paciente.

Grande parte da população relatou que os produtos vegetais utilizados são obtidos de suas próprias plantações caseiras. Neste estudo, não foi abordado um aspecto importante referente a identificação botânicas das espécies vegetais, que é necessário, considerando o fato de ser plantações caseiras e da indicação de familiares e amigos, como discutido acima. Sendo assim, são necessários estudos que discutam a identificação botânica, considerando que as plantas citadas nesse estudo não apresentam comprovação cientifica de uso, sendo que uti-

lizar a espécie errada pode representar um risco adicional ao paciente. No estudo de Elias $(2002)^{10}$, é relatado: a carência de comprovação científica, a falta de regulamentação, os efeitos adversos potenciais causados pela identificação equivocada das plantas.

A maioria dos entrevistados relatou a utilização da planta fresca com $61 \%$ das citações, seguida pelo sachê com 31\%, ambas com preparações de infusão. No estudo de Colômbia o método mais comum da ingestão foi na forma da planta fresca ${ }^{9}$. Também foi observado que os usuários utilizavam para o consumo xarope, pomadas e cápsulas a partir de produtos a base de plantas medicinais, mas com menos prevalência. Contudo, as infusões e os xaropes foram preparados nas residências dos usuários, sendo relatado pelos entrevistados o preparo de xarope para uso durante uma semana, armazenados na geladeira, sem cuidados de contaminação e, podendo ser preparados da maneira incorreta.

Diante disso, fica claro a necessidade da revisão do papel da equipe do Cacon na orientação e acompanhamento de paciente. Neste contexto, o farmacêutico deveria ter um contato mais direto com os usuários de plantas medicinais podendo orientar sobre: as indicações, o uso correto e salientar os efeitos adversos que o uso de terapias não convencionais.

\section{CONCLUSÃO}

Os resultados demonstram que parte dos pacientes oncológicos em tratamento quimioterápico faz uso de plantas medicinais ou fitoterápicos como forma complementar de tratamento. Os dados encontrados neste estudo são semeIhantes às informações de outros estudos, tanto brasileiros como internacionais sobre o uso de plantas medicinais e fitoterápicos complementares a quimioterapia. Observou-se semelhanças quanto as espécies utilizadas e ao seu uso ser baseado no conhecimento tradicional, sem a orientação dos profissionais da saúde. Mostrando a necessidade de uma melhor comunicação entre o profissional de saúde e o paciente, com destaque aos oncológicos, uma vez que o uso 
inadequado de plantas nesta população, podem desencadear riscos maiores à saúde.

Observa-se que os usuários de planta entrevistados neste estudo carecem de informações acerca do uso e dos riscos, sendo que este dado pode ser um alerta aos órgãos sanitários, considerando que mesmo que haja a proibição da venda do produto em estabelecimentos, a utilização foi verificada pela população do estudo. Isso é possível pela população que produzem as plantas medicinais em suas residências. Mostrando ser este um grave problema de saúde pública, uma vez investe-se em novos tratamentos alopáticos, caros, e não se orienta quanto ao uso de plantas e fitoterápicos que podem estar pre- judicando o tratamento e a saúde de pacientes tão frágeis como os oncológicos.

Destaca-se que algumas plantas citadas no presente estudo, como aloe vera e Euphorbia tirucalli são contra indicadas pela ANVISA para pacientes oncológicos, por meio que resoluções, que, embora em vigor, não chegaram ao conhecimento da população, principalmente pela falta de assistência a estes usuários nos serviços de saúde, mostrando a necessidade de rever as funções dos profissionais da saúde que integram as equipes multidisciplinares dos serviços de oncologia do Brasil, assim como fomentar atividades educativas com essa população.

\section{REFERÊNCIAS}

1. Schenkel EP, Mengue SS, Petrovick PR. Cuidado com medicamentos Florianópolis: UFSC; 2012. 256p.

2. Casarin ST, Heck RM, Schwartz E. O uso de práticas terapêuticas alternativas, sob a ótica do paciente oncológico e sua família. Fam. Saúde Desenv 2005 jan./abr;1(7):24-31.

3. Cassileth BR, Chapman CC. Alternative and complementary cancer therapies. Cancer 1996;77(6):1026-34

4. BRASIL. Ministério da Saúde. Secretaria de Ciência, Tecnologia e Insumos Estratégicos. Departamento de Assistência Farmacêutica e Insumos Estratégicos. Programa Nacional de Plantas Medicinais e Fitoterápicos - Brasília: Ministério da Saúde, 2009. 140p.

5. BRASIL. Agência Nacional de Vigilância Sanitária. Resolução - RDC No 10, de 9 de Março de 2010. Dispõe sobre a notificação de drogas vegetais junto à Agência Nacional de Vigilância Sanitária (ANVISA) e dá outras providências. Acesso em: 03 de janeiro de 2014. Disponível em: http://www.anvisa.gov.br/medicamentos/fitoterapicos/definicao.htm.

6. BRASIL. Agência Nacional de Vigilância S

anitária. Formulário de Fitoterápicos da Farmacopeia Brasileira / Agência Nacional de Vigilância Sanitária. Brasília: ANVISA, 2011. 126p.

7. Vanini M, Barbieri RL, Heck RM, Schwartz E. Uso de plantas medicinales por pacientes oncológicos y familiares em um centro de redioterapia. Enferméria global. 2011;21, p.1-6.

8. Araújo EC, Oliveira RAG, Coriolano AT, Araújo EC. Uso de plantas medicinais pelos pacientes com câncer de hospitais da rede pública de saúde em João Pessoa (PB), Revista Espaço Para a Saúde, 2007 jun; 2(8):44-52.

9. Diaz LA, Torregrosa L, Benítez L, Mercado M, Fiorentino S. Plant-based Complementary and alternative medicine used by breast cancer patients at the Hospital Universitario San Ignacio in Bogotá, Colombia. Universitas Scientiarum 2012;17(3):291-302.

10. Elias MC, Alves E. Medicina não-convencional: prevalência em pacientes oncológicos Revista Brasileira de Cancerologia, 2002,48(4):523-32.

11. Myers SP, Cheras PA. The other side of the coin: safety of complementary and alternative medicine. Medical Journal of Australia, 2004;181(4):222-5.

12. Schwambach KH, Amador TA. Estudo da Utilização de Plantas Medicinais e Medicamentos em um Município do Sul do Brasil. Latin American JournalofPharmacy. 2007; 26(4):602-8.

13. BRASIL. Ministério da Saúde. Agência Nacional de Vigilância Sanitária. Instrução Normativa № 5 , de 31 de março de 2010.

14. BRASIL. Ministério da Saúde. Secretaria de Ciência, Tecnologia e Insumos Estratégicos. Departamento de Assistência Farmacêutica e Insumos Estratégicos. Relação Nacional de Medicamentos Essenciais: Rename - 8. ed. - Brasília: Ministério da Saúde, 2013. 200p.

15. Simões CMO, Schenkel EP, Gosman G, et al. (Org.) Farmacognosia: da planta ao medicamento. 6 ed. Porto Alegre: Editora da UFSC e UFRGS Editora., 2007.

16. Jacondino CB, Amestoy SC, Thofehrn MB. A utilização de terapias alternativas por pacientes em tratamento quimioterápico. Revista Cogitare. 2008; 22(3):61-6. 
17. BRASIL. Agência Nacional de Vigilância Sanitária. Resolução no 2.744, de 16 de junho de 2010. Proibido o comércio de produtos sem registro, 2006. Acesso em: 25 de Janeiro de 2014. Disponível em: <http://www.anvisa.gov.br/divulga/ noticias/2006/140806_2.htm>.

18. BRASIL. Informe Técnico nº. 47, de 16 de novembro de 2011b. Agência Nacional da Vigilância Sanitária. Disponível em: <http://portal.anvisa.gov.br/wps/wcm/connect/c66ea5804924c8f49d829f14d16287af/Informe_Tecnico_n_47_de_16_ de_novembro_de_2011.pdf?MOD=AJPERES>. Acesso em: 25 de Janeiro de 2014.

19. BRASIL. Ministério da Saúde. Resolução RE No 2.917, de 6 de julho de 2011c. Acesso em: 20 de janeiro de 2014. Disponível em: http://bvsms.saude.gov.br/bvs/saudelegis/anvisa/2011/res2917_06_07_2011.html

20. Rosa C, Câmara SG, Béria JU. Representações e intenção de uso da fitoterapia na atenção básica à saúde. Revista Ciência \& Saúde Coletiva, 2011;1(16):311-8. 